\title{
Calnexin is necessary for $T$ cell transmigration into the central nervous system
}

\author{
Joanna Jung, ${ }^{1}$ Paul Eggleton, ${ }^{2,3}$ Alison Robinson, ${ }^{1}$ Jessica Wang, ${ }^{1}$ Nick Gutowski, ${ }^{2}$ Janet Holley, ${ }^{2}$ \\ Jia Newcombe, ${ }^{4}$ Elzbieta Dudek, ${ }^{1}$ Amber M. Paul, ${ }^{5,6}$ Douglas Zochodne, ${ }^{6}$ Allison Kraus, ${ }^{1}$ \\ Christopher Power, ${ }^{5,6}$ Luis B. Agellon, ${ }^{7}$ and Marek Michalak ${ }^{1,5}$ \\ 'Department of Biochemistry, University of Alberta, Edmonton, Alberta, Canada. ${ }^{2}$ University of Exeter Medical School, \\ Exeter, Devon, United Kingdom. ${ }^{3}$ UCB Pharma, Slough, Berkshire, United Kingdom. ${ }^{4}$ NeuroResource, UCL Institute of \\ Neurology, University College London, London, United Kingdom. ${ }^{5}$ Multiple Sclerosis Centre and ${ }^{6}$ Department of Medicine \\ (Neurology), University of Alberta, Edmonton Alberta, Canada. ${ }^{7}$ School of Human Nutrition, McGill University, \\ Ste. Anne de Bellevue, Quebec, Canada.
}

In multiple sclerosis (MS), a demyelinating inflammatory disease of the CNS, and its animal model (experimental autoimmune encephalomyelitis; EAE), circulating immune cells gain access to the CNS across the blood-brain barrier to cause inflammation, myelin destruction, and neuronal damage. Here, we discovered that calnexin, an ER chaperone, is highly abundant in human brain endothelial cells of MS patients. Conversely, mice lacking calnexin exhibited resistance to EAE induction, no evidence of immune cell infiltration into the CNS, and no induction of inflammation markers within the CNS. Furthermore, calnexin deficiency in mice did not alter the development or function of the immune system. Instead, the loss of calnexin led to a defect in brain endothelial cell function that resulted in reduced $\mathrm{T}$ cell trafficking across the blood-brain barrier. These findings identify calnexin in brain endothelial cells as a potentially novel target for developing strategies aimed at managing or preventing the pathogenic cascade that drives neuroinflammation and destruction of the myelin sheath in MS.

Conflict of interest: The authors have declared that no conflict of interest exists.

Submitted: October 31, 2017 Accepted: January 30, 2018 Published: March 8, 2018

Reference information: JCI Insight. 2018;3(5):e98410. https:// doi.org/10.1172/jii.insight.98410.

\section{Introduction}

Multiple sclerosis (MS) is a leading cause of disability in young adults and affects over 2.5 million people worldwide $(1,2)$. MS is an inflammatory demyelinating disease of the CNS mediated by autoreactive T cells (3). In MS and in its animal model (experimental autoimmune encephalomyelitis; EAE), circulating immune cells access the CNS across the blood-brain barrier to cause inflammation, myelin destruction, and neuronal damage, all of which set the stage for the development of the clinical manifestations of this disabling/progressive disease. The pathology of MS includes the presence of multiple demyelinated plaques in the CNS, the presence of inflammatory immune cells in the brain, loss of oligodendrocytes, and axonal demyelination and degeneration.

Blood-brain barrier dysfunction is a hallmark of many CNS disorders, including MS $(4,5)$. It consists of a complex multicellular structure composed of a highly specialized and tightly sealed monolayer of brain endothelial cells buttressed by pericytes in the basal membrane, perivascular macrophages, and astrocyte foot processes $(4,5)$. The blood-brain barrier restricts the transfer of molecules to the CNS parenchyma, but when disrupted - for example due to inflammation or stroke - neuronal functions can be compromised (6). Brain endothelial cells achieve their highly selective permeability through a junctional complex by using both tight junctions and adherens junctions. Tight junctions between endothelial cells are principally responsible for the barrier function. Under healthy conditions, CNS endothelial cells express low levels of leukocyte adhesion molecules, such as vascular cell adhesion molecule 1 (VCAM1) and intercellular adhesion molecules (ICAMs; ICAM1 and -2) but are expressed highly in the vasculature during $\mathrm{EAE}$ and MS to promote T cell influx into the brain parenchyma (7). T cell/endothelial cell interactions are mediated by adhesion molecules and chemokines and their respective chemokine receptors (4). Tight junction proteins (platelet endothelial cell adhesion molecule [PECAM], junctional adhesion molecule [JAM], 
occludin, claudins, and cadherin) regulate paracellular flux and prevent molecules/cells from translocating across the blood-brain barrier $(4-6,8)$. Integrin activation leads to enhanced affinity of the adhesion molecule for its endothelial blood-brain barrier ligands including VCAM1, ICAM1, and P-selectins to support lymphocyte exit from the blood flow by extravasating through the vascular endothelial cell wall, a process of transendothelial migration (4-6).

The ER is an elaborate membrane system that houses a variety of cellular functions that are critical for the maintenance of cellular homeostasis; synthesis of membrane-associated, luminal, and secreted proteins; correct folding and posttranslational modification of proteins and glycoproteins; lipid and steroid synthesis; and $\mathrm{Ca}^{2+}$ signaling and storage (9-11). To perform these diverse functions, the ER engages a wide assortment of multifunctional integral membrane and luminal chaperones, including calnexin, an ER integral membrane protein (12). This protein is involved in the folding and quality control of membrane-associated and secreted glycoproteins (10). Elimination of calnexin function has diverse effects in different organisms. Calnexin deficiency is lethal in Schizosaccharomyces pombe but not in Saccharomyces cerevisiae, Dictyostelium (13), or Caenorhabditis elegans (14). Interestingly, the loss of calnexin affects phagocytosis in Dictyostelium (15) and endocytosis in mammalian cells (16). Calnexin is highly expressed during the development of the nervous system $(17,18)$, and in mice, calnexin deficiency causes a peripheral axonal dysmyelinating phenotype involving reduced peripheral nerve conduction velocities (18-20).

What is the calnexin link between the immune system and the nervous system? To answer this question, we examined the involvement of calnexin in the pathogenesis of MS. In this study, we discovered that calnexin is highly abundant in the brains of MS patients, while deficiency of calnexin in mice confers resistance to EAE. Although MS is regarded as a progressive autoimmune disease of the CNS, we did not find evidence indicating that calnexin deficiency in mice causes impaired development or function of the immune system. In contrast, we observed that the loss of calnexin caused a defect in CNS endothelial cell function that resulted in resistance of the blood-brain barrier to circulating $\mathrm{T}$ cell infiltrations. These findings identify a link between calnexin expression in CNS endothelial cells and the pathogenic cascade that drives neuroinflammation and destruction of the myelin sheath seen in MS.

\section{Results}

Calnexin is upregulated in brain samples of MS patients. Clinical symptoms of MS result from axonal and neuronal dysfunction and injury in human brain tissue. MRI patient brain scans characteristically show white matter lesions on FLAIR and T2-weighted sequences (Figure 1A). Lesions often occur in the periventricular region and juxtacortical white matter, sites enriched with venules, where blood-brain barrier breakdown frequently occurs. We discovered a high abundance of calnexin immunoreactivity (Figure 1B, left column) and immune cell infiltration (Figure 1B, right column, Oil Red O-stained) in MS brain sections, including acute, subacute, and chronic demyelinating lesions, as well as normal-appearing white matter (NAWM). Calnexin abundance in acute and chronic lesions was greater compared with control, NAWM, and subacute brain lesion tissue. Flow cytometric analysis also revealed high abundance of calnexin in MS brain endothelial cells (Figure 1C) indicating that calnexin was expressed in human CNS endothelial cells. This was further supported by immunostaining of human brain microvascular capillaries in control (non-MS) tissue versus acute and chronic demyelinating lesions (Figure 1C).

Calnexin-deficient mice are resistant to induction of EAE. To test whether high abundance of calnexin is important to MS pathogenesis, we induced EAE in calnexin-deficient mice using myelin oligodendrocyte glycoprotein $\left(\mathrm{MOG}_{35-55}\right.$ ) inoculation in complete Freund's adjuvant (CFA). Within the first 2 weeks of injection, the WT mice - as well as mice with only 1 functional calnexin allele (heterozygotes, Can $^{+/-}$) - developed typical neurobehavioral deficits associated with EAE induction (Figure 2A). Surprisingly, calnexin-deficient mice ( Canx $^{--}$) exhibited no signs of EAE throughout the disease course (23 days) (Figure 2A). Consistent with these neurobehavioral features, histologic evaluation of the CNS tissue at 14 days after immunization revealed dramatic differences between calnexin-deficient animals and WT littermates (Figure 2B and Figure 3). In the EAE WT animals, there was significant infiltration of leukocytes into the white matter of the spinal cord (Figure $2 \mathrm{~B}$ and Figure $3 \mathrm{~A}$ ). In contrast, spinal cords from calnexin-deficient mice immunized with the $\mathrm{MOG}_{35-55}$ peptide showed no evidence of immune cell infiltration (Figure $2 \mathrm{~B}$ and Figure 3A). Immunostaining of WT and calnexin-deficient lumbar spinal cord section for markers of $\mathrm{T}$ cells, macrophages, and microglia (CD3e, GFAP, IBA-1, F4/80) also showed no evidence of infiltration of these cells in calnexin-deficient EAE mice (Figure 3, A and B). Next, we analyzed the abundance of tran- 
A

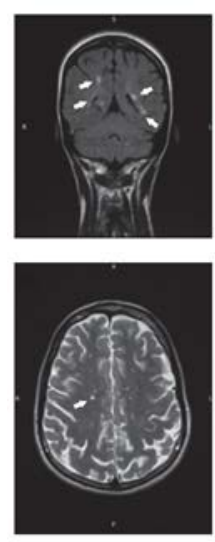

B

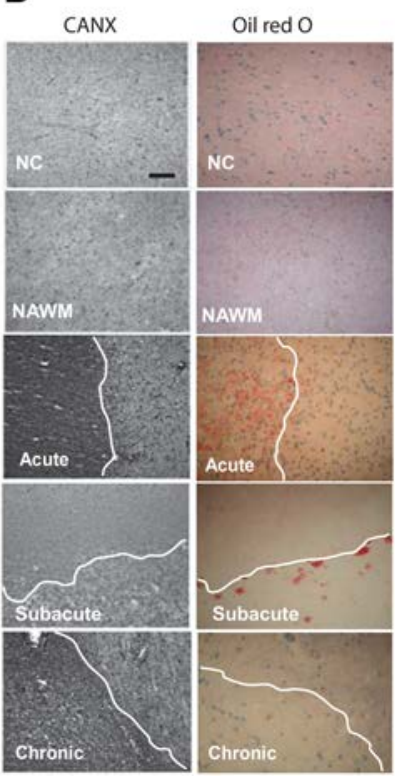

C
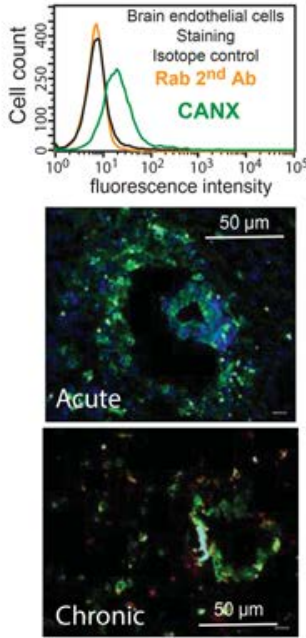
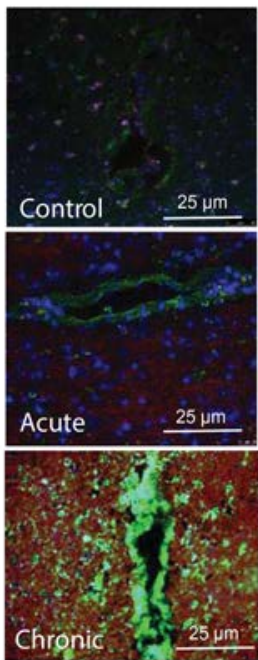

Figure 1. High abundance of calnexin in acute and chronic lesions in brain of multiple sclerosis patients. (A) MRI scans of a patient with multiple sclerosis (MS) demonstrating high-signal white matter lesions on FLAIR (top) and T2-weighted (bottom) sequences. Lesions depicted by arrows. (B) 3,3'-Diaminobenzidine (DAB) nickel chloride staining for calnexin (CANX) in non-MS control (NC), normal-appearing white matter (NAWM) MS brain, acute lesions (acute), subacute lesions (subacute), and chronic lesions together with Oil Red O/hematoxylin staining of parallel $10-\mu \mathrm{m}$ sections of MS tissue, as previously described (44). Lesions are demarcated by a white lined. Scale bar: $25 \mu \mathrm{m}$. (C) FACS analysis of CANX in the isolated human brain endothelial cells. Immunofluorescence staining for calnexin in the blood vessels of (top right) non-MS individual and capillaries located in the acute and chronic lesions of MS patient tissue, as previously described (44).

scripts encoding inflammation-specific genes in the spinal cords from control and EAE-induced mice (Figure 3C). The mRNAs for $\mathrm{T}$ cell receptor $\mathrm{CD} 3 \varepsilon$, early activation marker; $\mathrm{CD} 69$, marker of inflammation; macrophage/microglia activation marker F4/80; GFAP; and cytokines IL-17, IL-1 $\beta$, IL-6, and GM-CSF were dramatically increased in WT mice with induced EAE (Figure 3C). In agreement with histological analysis (Figure 2B and Figure 3A) of CNS tissue from calnexin-deficient animals with induced EAE, there was no induction of mRNA encoding any of the tested inflammation markers (Figure 3C).

To test the specificity of calnexin in conferring resistance of the $\mathrm{Canx}^{-1-}$ mice to the EAE induction, we generated $\mathrm{Canx}^{-/}$mice reconstituted with a transgene ( $\mathrm{Tg}$-CanxFL) that directs the ubiquitous expression of the full-length calnexin protein. Immunoblot analysis of cells isolated from the Canx ${ }^{1-}$-Tg-CanxFL mice confirmed the abundance of full-length calnexin protein and calnexin mRNA in the reconstituted mice (Figure 4, A and B). The Canx ${ }^{/-}-\mathrm{Tg}$-CanxFL exhibited normal motor function phenotype (18) with respect to conduction velocity analyses of peripheral motor and sensory axons (Figure 4, C and D). Importantly, the susceptibility of the Canx ${ }^{-/}$-TG-CanxFL to induction of EAE was similar to that observed in WT or heterozygote animals (Figure 2A). Taken together, these findings demonstrate that global deletion of the calnexin gene in mice was protective against development of EAE and was associated with the impaired transmigration of encephalitogenic lymphocytes into the CNS parenchyma of Canx $x^{-1}$ mice. Furthermore, resistance to EAE required calnexin absence because susceptibility to EAE was restored by expression of the recombinant full-length calnexin protein in calnexin-deficient mice.

Peripheral immune response was not affected by the loss of calnexin. Next, we asked whether calnexin deficiency impacted the development and function of the peripheral immune system and, hence, resistance of the calnexin-deficient mice to EAE induction. MS is an autoimmune disease in which antibodies and T cells targeting myelin antigens are present in the blood (21). The induction of EAE by immunization of mice with $\mathrm{MOG}_{35-55}$ leads to the appearance of anti-MOG antibodies and encephalogenic $\mathrm{T}$ cells in blood from immunized mice upon onset of the disease (22). Consequently, secreted anti-MOG antibodies and T cells are pathogenic and contribute to demyelinating EAE episodes. Sera from WT and Canx $x^{-1-}$ mice treated 


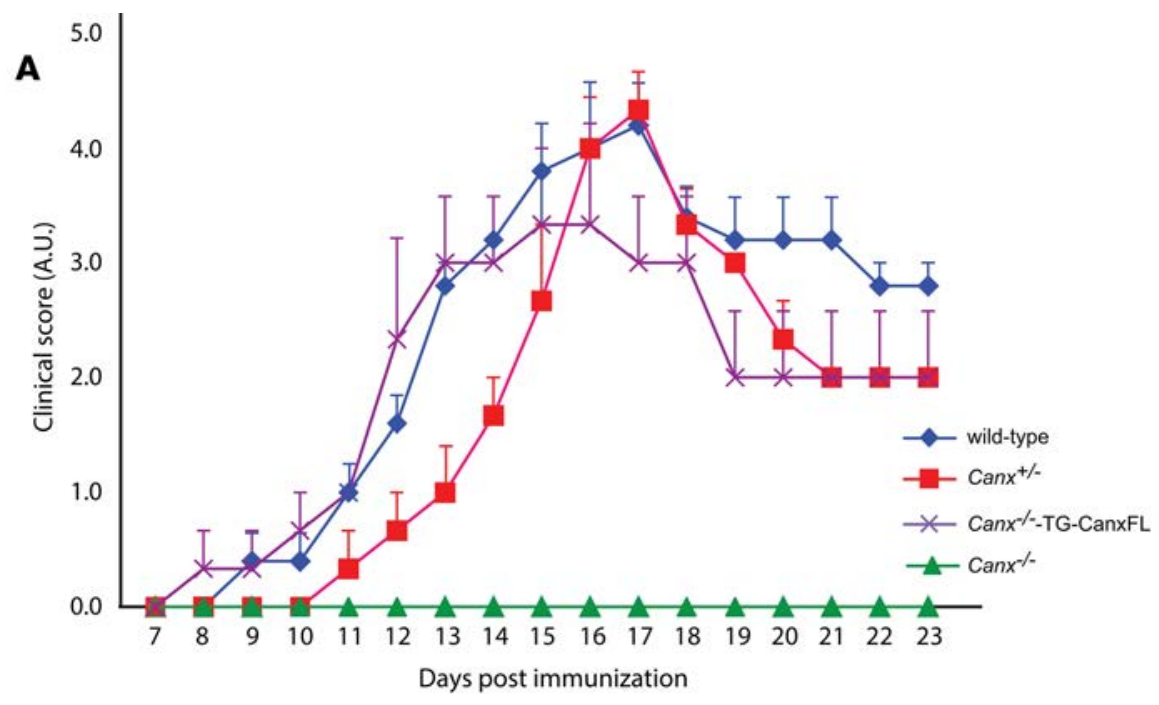

B
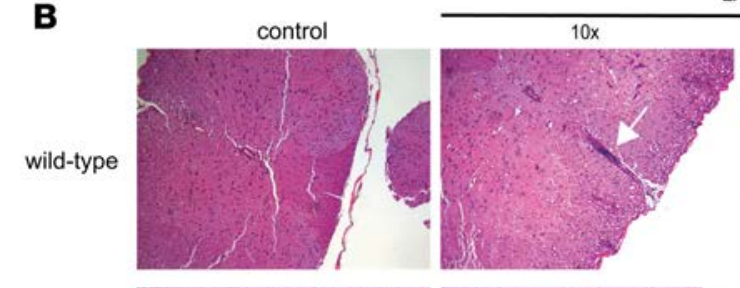

EAE
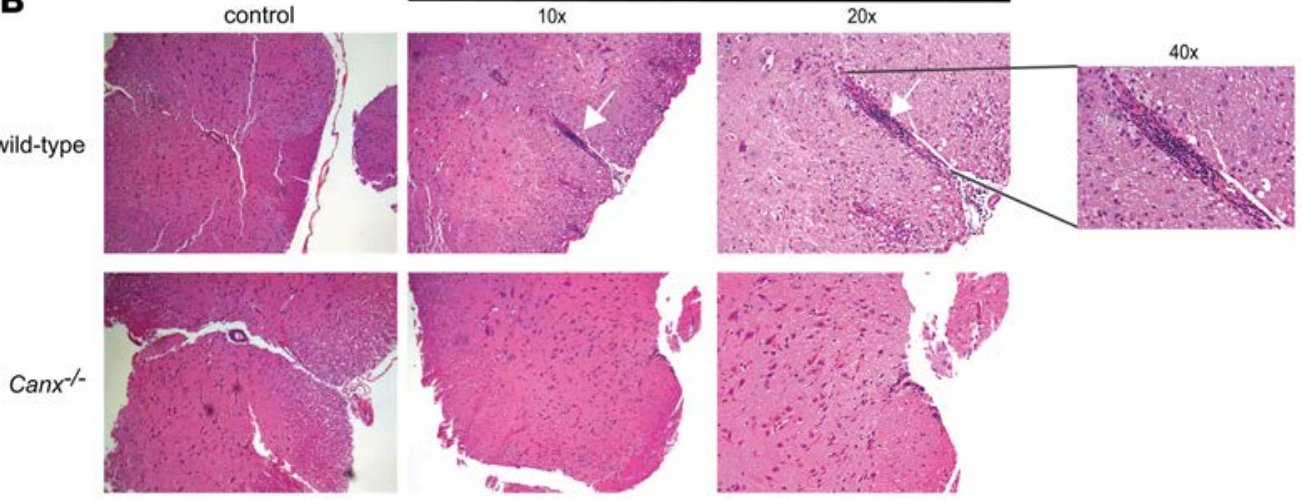

Figure 2. Calnexin-deficient mice exhibit no clinical symptoms of EAE or mononuclear cell infiltration of CNS. (A) Graph representing daily clinical scores. EAE was induced by $\mathrm{MOG}_{35-55}$ immunization of WT, Canx ${ }^{+/-}$(heterozygote),

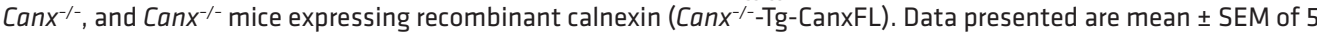
independent experiments (20 animals per group) with 5 replicates. The means were compared using unpaired 2-tailed Student's $t$ test. (B) Histology of spinal cord tissue from control and EAE WT and Canx ${ }^{-1-}$ mice. H\&E staining; 10x, 20x, and 40x magnifications are shown. The arrows indicate accumulation of mononuclear cells in the white matter of the spinal cord. No accumulation of mononuclear cells was observed in similar sections from the Can $x^{-/-}$mice. Data shown is representative of 3 mice per experimental group.

with $\mathrm{MOG}_{35-55}$ were collected and used as the primary antibodies for immunoblot analysis of brain samples (Figure 5, A and B). Sera from non-EAE mice were used as a negative control, and purified recombinant MOG protein (recMOG; AnaSpec, 14.3-kDa) was used as a positive control (Figure 5, A and B). Immunoblot analysis indicated that both WT and calnexin-deficient mice immunized with $\mathrm{MOG}_{35-55}$ produced anti-MOG antibodies (Figure 5, $\mathrm{A}$ and $\mathrm{B}$ ), indicating that, with $\mathrm{MOG}_{35-55}$ antigen presentation, the production and secretion of $\gamma$ antibodies were not affected in the absence of calnexin. Furthermore, we found no difference in the number of $\mathrm{CD} 4^{+} / \mathrm{CD}^{+}$and $\mathrm{CD}^{+} / \mathrm{CD}^{+}$cells and MHC class I and MHC class II expression between WT and calnexin-deficient splenocytes challenged with $\mathrm{MOG}_{35-55}$ (Supplemental Figure 1; supplemental material available online with this article; https://doi.org/10.1172/jci.insight.98410DS1). We concluded, therefore, that the functional adaptive immunity was not compromised in Can $x^{-1-}$ mice and did not contribute to EAE resistance exhibited by $\mathrm{Canx}^{-1-}$ mice.

In agreement with anti-MOG immunoblotting results (Figure 5, A and B), multiplex array analysis of cytokine production in the periphery showed no differences between WT and Canx $x^{-1}$ mice (Figure 5C). IL-17 is recognized to play a role in the development and progression of EAE and MS (23). Both stimulated WT and Canx $x^{-1}$ T cells and T cells isolated from EAE-induced animals secreted IL-17 (Figure 5, D and E). Most importantly, there were similar differences in IL-17 secretion between stimulated WT and Can $x^{-1-} \mathrm{T}$ 

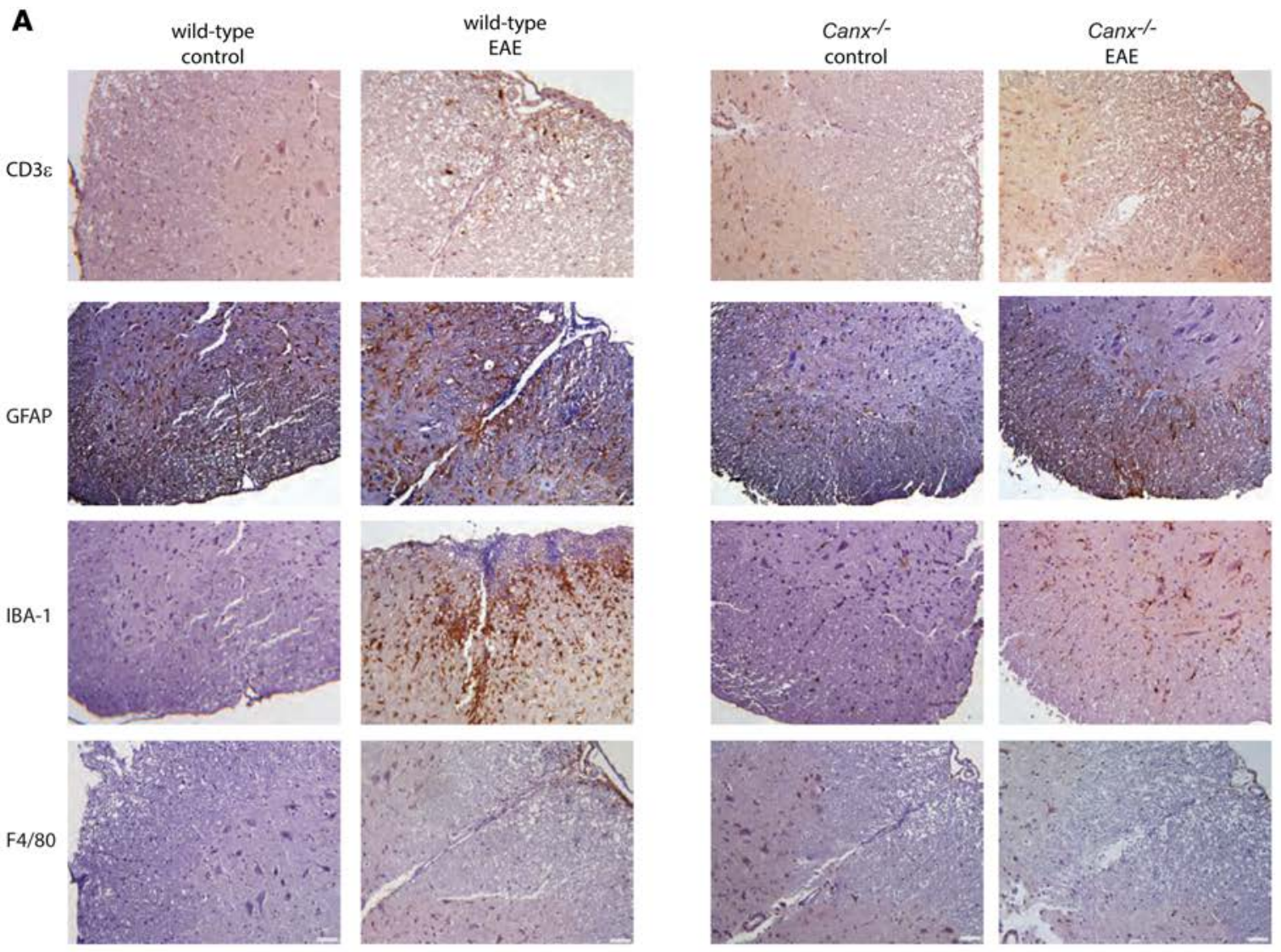

B
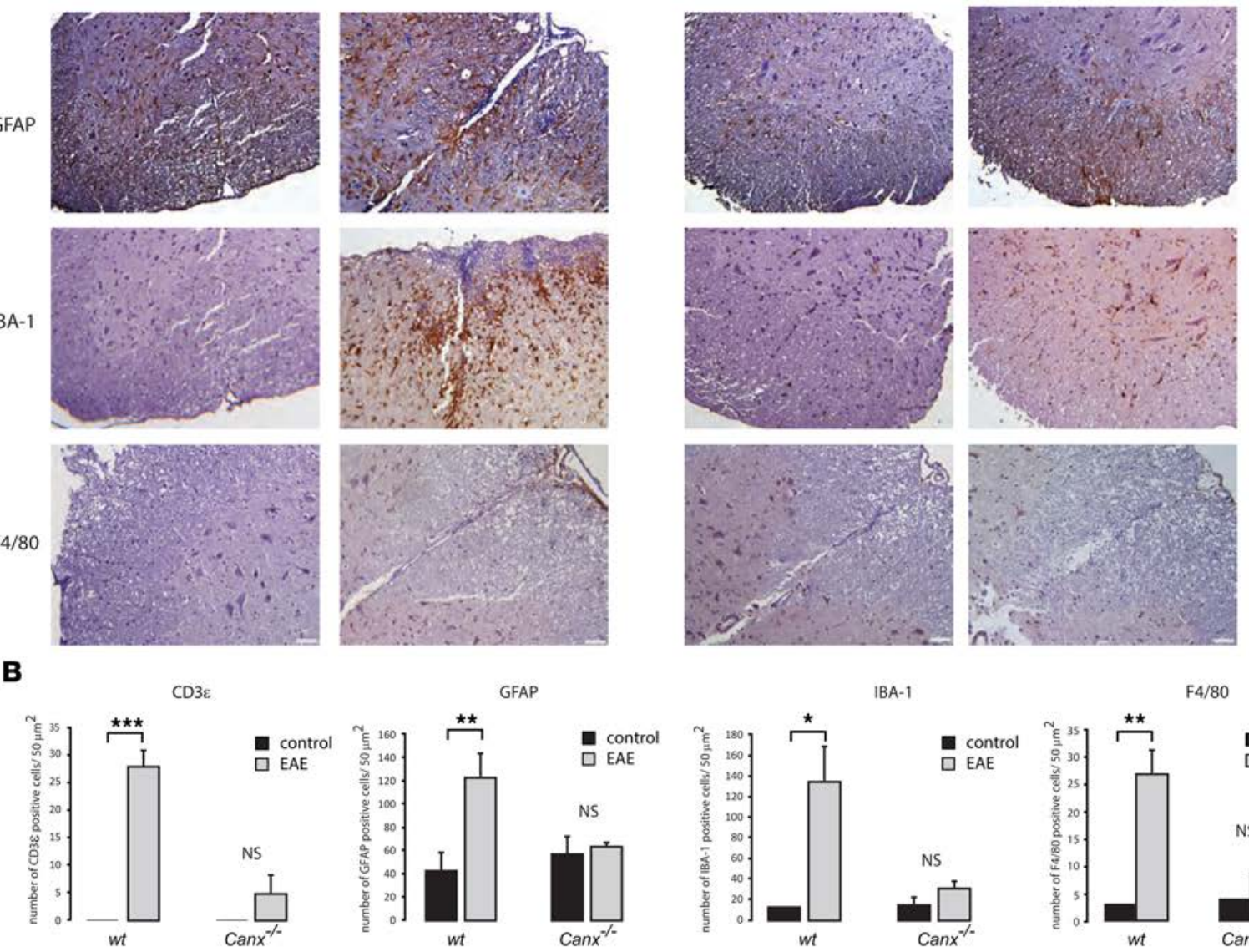

C
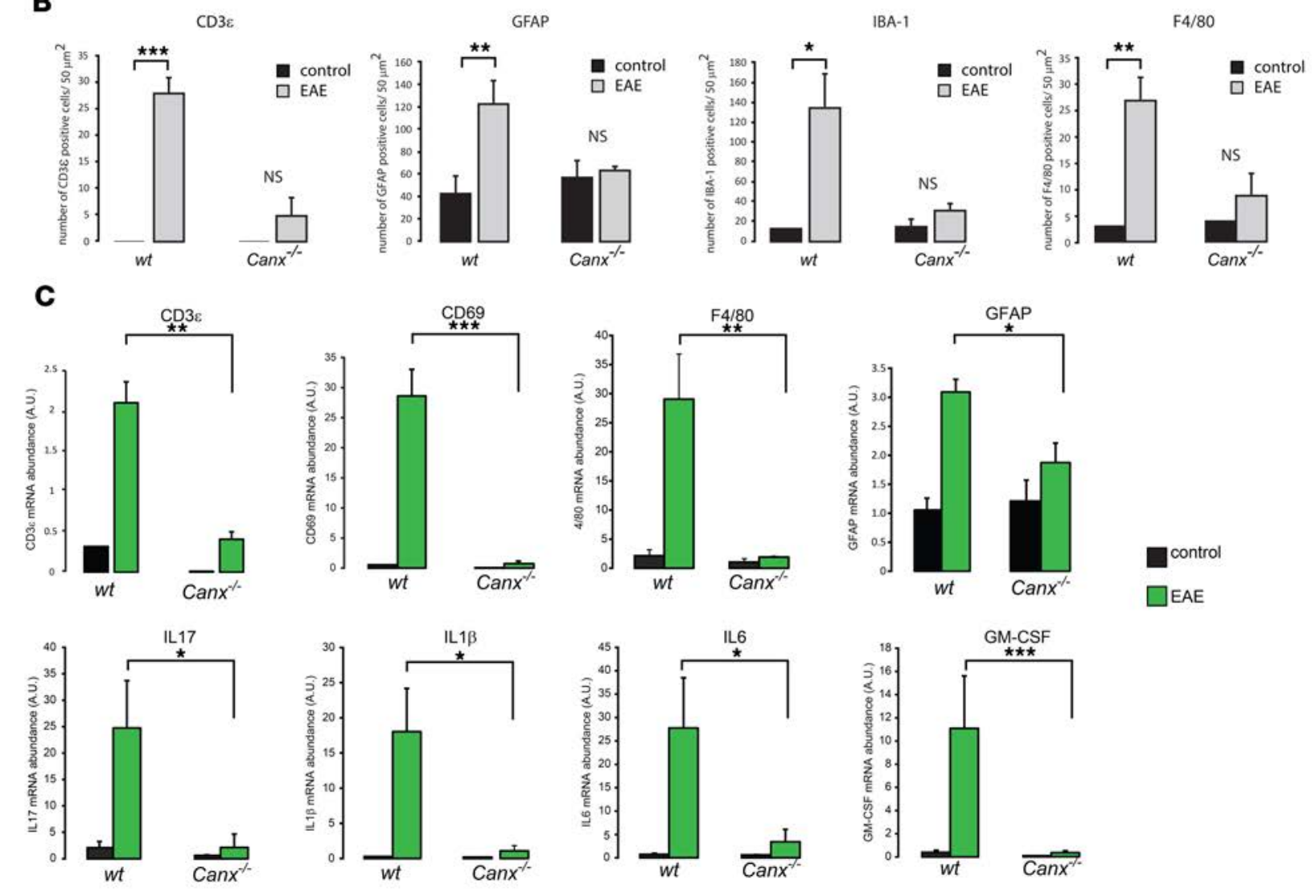
Figure 3. Calnexin-deficient mice exhibit no immune cell infiltration and suppressed inflammatory gene expression. (A) Immunostaining of lumbar spinal cord sections for selected immune cell infiltrates in WT and calnexin-deficient control or EAE mice. Magnification, 20x; scale bars: $50 \mu \mathrm{m}$. (B) Quantitative analysis of CD3E-, GFAP-, IBA-1-, and F4/80-positive cells ( $n=3$ mice per group). The number of positive cells was quantified in 5 randomly chosen fields $\left(50 \mu \mathrm{m}^{2}\right)$ in each section of white matter. Nonpaired 2-tailed Student's $t$ test was used to determine statistical analysis. ${ }^{*} P \leq 0.02 ;{ }^{* *} P \leq 0.006$; ${ }^{* * *} P$ $\leq$ 0.0006. (C) Spinal cord inflammation was assessed by quantitative PCR of inflammation markers. ${ }^{*} P \leq 0.05$; ${ }^{* *} P \leq 0.01$; ${ }^{* * *} P \leq 0.001$. Data presented are mean \pm SEM of 3 independent experiments, 3 mice per group. The means were compared using unpaired 2-tailed Student's $t$ test.

cells (Figure 5, D and E). Taken together, these findings indicated that the EAE resistance observed in calnexin-deficient animals was not caused by a reduced number of circulating activated immune cells, by their ability to produce and secrete proinflammatory cytokines, by altered function of antigen presenting cells, or by the ability of B cells to produce and secrete antibodies.

Targeted calnexin deficiency in T cells did not protect mice from clinical signs of EAE. To further evaluate the involvement of $\mathrm{T}$ cells in induction of $\mathrm{EAE}$, we generated mice with flox sequences incorporated in the calnexin gene ( $\left.C a n x^{f / f f}\right)$ and crossed them with mice expressing the Cre recombinase under the control of the Lck proximal promoter $\left(\mathrm{Lck}-\mathrm{Cr} \mathrm{e}^{+}\right.$mice), which is predominantly active in T cells-(24-26). This cross produced $C a n x^{f l f l} / L c k-C r e^{+}$mice with a deletion of $794 \mathrm{bp}$ from exons 3 and 4 of the Canx gene in T cells (27). The specific inactivation of the Canx gene in T cells of Canx $x^{f / f t} / L c k-C r e^{+}$mice was confirmed by immunoblot analysis of the thymus (Figure 6A). Remarkably, FACS analysis revealed that the thymus, spleen, and peripheral $\mathrm{T}$ cell populations were not affected by the targeted inactivation of the calnexin gene in the T cells of Canx ${ }^{f l f t} / L c k-C r e^{+}$mice (Figure 6B) and demonstrated that the absence of calnexin had no significant impact on the development of the immune system. Next, we inoculated WT and Canx ${ }^{f / f l} / L c k-C r e^{+}$mice with $\mathrm{MOG}_{35-55}$. Like WT mice, Canx $x^{f / f l} / L c k-C r e^{+}$mice developed EAE symptoms (Table 1), indicating that calnexin deficiency in T cells specifically did not interfere with the recruitment of encephalitogenic lymphocytes into the CNS and the induction of EAE.

Transendothelial migration of T cells across the brain endothelium was inhibited in the absence of calnexin. A pivotal feature of MS pathogenesis is that proinflammatory $\mathrm{T}$ cells in the blood cross the blood-brain barrier, resulting in focal inflammation and demyelination. Thus, we asked if the function of the blood-brain barrier was altered in the absence of calnexin and whether this was responsible for protection of $\mathrm{Canx}^{-1-}$ mice against EAE induction. To address this question, we used a mouse brain endothelial cell line, bEND.3 $(28,29)$, which serves as a cell culture model for studies of the blood-brain barrier endothelium. First, we found that cytokine-stimulated bEND.3 CNS endothelial cells displayed an increased abundance of calnexin immunoreactivity (Figure 7A). This finding recapitulated our observations in human brain sections from MS patients (Figure 1) and supported the notion that calnexin expression might be increased in the blood-brain barrier endothelial cells of $\mathrm{MOG}_{35-}$ ${ }_{55}$-treated mice. Next, we used an in situ gene-editing technique to generate calnexin-deficient bEND.3 cells (Canx $x^{-1-}$-bEND.3) (Figure 7B and Supplemental Figure 2). WT bEND.3 cells and Canx $x^{-/}-\mathrm{bEND} .3$ cells showed a similar response to IL-1 $\beta$, TNF- $\alpha$ or IFN- $\gamma$ cytokine with respect to expression of VCAM1 and ICAM1 (Figure 7C). Cytokine stimulation of WT and calnexin-deficient bEND. 3 cells also resulted in increased abundance of tight junction molecules PECAM and JAM-A (Figure 7D). Next, using a monolayer of bEND.3 cells as a tissue culture model of the endothelium, we tested whether absence of calnexin impacted on blood-brain barrier endothelial cells function with respect to $\mathrm{T}$ cell transendothelial migration. WT stimulated $\mathrm{T}$ cells were added to the upper chamber containing a layer of cytokine-stimulated WT or Can $x^{-/}-\mathrm{bEND} .3$ cells, followed by FACS analysis of the T cells crossing the endothelial cell layer into the bottom chamber (Figure 7E). As expected, there was significant transmigration of WT T cells across cytokine-stimulated WT bEND.3 cells (Figure 7E). In stark contrast, transendothelial migration of T cells across cytokine-stimulated Can $x^{-/-}$-bEND. 3 cells was significantly reduced (Figure 7E), demonstrating that the impairment of calnexin function in CNS endothelial cells in Canx ${ }^{\prime-}$ mice underlies the resistance of these mice to EAE induction.

\section{Discussion}

As a molecular chaperone, calnexin has been implicated in immunity because of its capacity to regulate the folding and assembly of the MHC class I complex or folding of the T cell receptor (30). During development, calnexin is highly expressed in the nervous system, and elimination of calnexin in mice results in peripheral axonal dysmyelination, resulting in reduced nerve conduction velocities (18-20). These findings revealed that the protein is important for the development and function of the nervous system. Here, we discovered a high abundance of calnexin protein in the brains of MS patients, suggesting that increased 


\section{A}

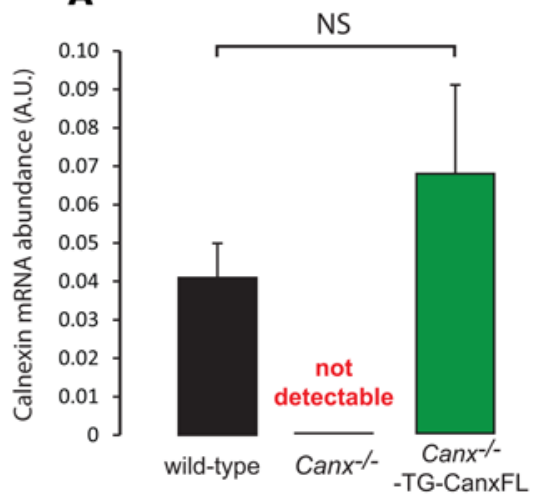

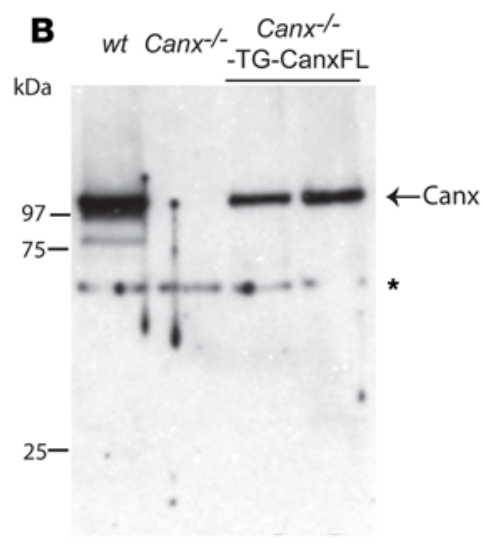

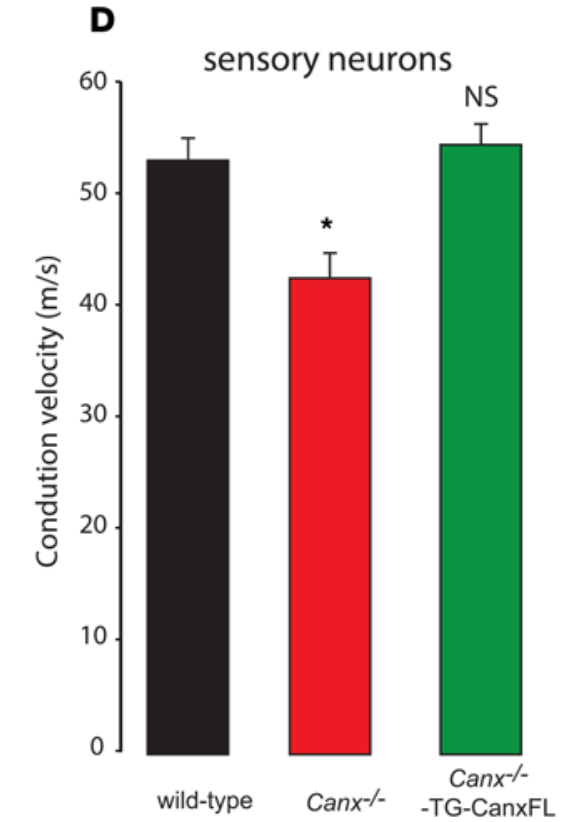

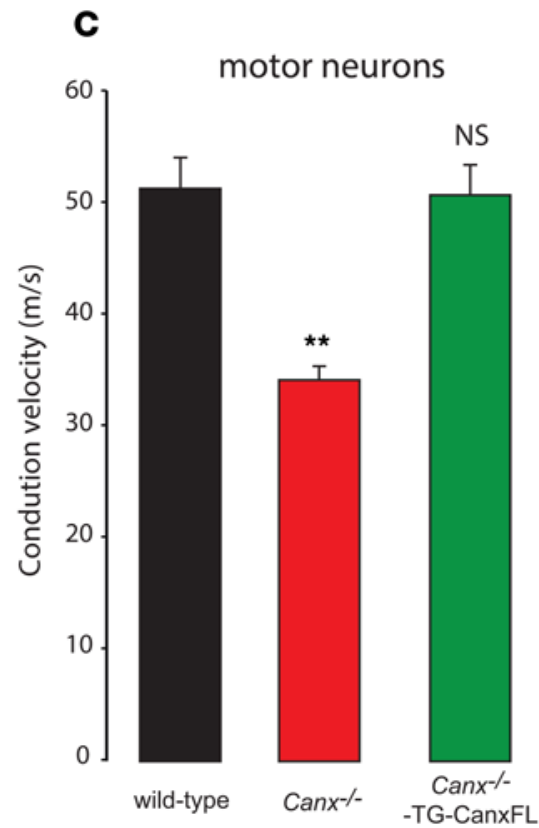

Figure 4. Transgenic Canx ${ }^{-/}$mice expressing full-length calnexin protein. (A) Abundance of calnexin mRNA in fibroblast cell lines isolated from WT and calnexin-deficient transgenic animals expressing full-length calnexin (Canx $x^{-/-}-\mathrm{Tg}^{-}$ CanxFL). Data are representative of 3 biological replicates. Canx, calnexin. Statistical analysis was done by unpaired 2-tailed Student's $t$ test. (B) Immunoblot analysis of fibroblasts isolated from WT, calnexin-deficient (Canx $x^{--}$), or Canx $x^{-1-}$-Tg-CanxFL. Immunoblot was probed with anti-calnexin antibodies. Asterisk represents nonspecific immunoreactive protein band. (C and D) Conduction velocity of motor (C) or sensory (D) neurons in WT, Canx $x^{-1-}$, and Canx $x^{-1-}$ Tg-CanxFL. ${ }^{*} P=0.013 ;{ }^{* *} P=0.0051 ; n=6$. Statistical analysis was done using unpaired 2 -tailed Student's $t$ test. See complete unedited blots in the supplemental material.

calnexin expression contributes to the pathogenesis of MS. The relevance of CNS calnexin to the occurrence of MS is demonstrated by our finding that mice lacking calnexin become resistant to EAE, despite the appearance of anti-MOG antibodies in the blood of calnexin-deficient mice immunized with $\mathrm{MOG}_{35-55}$ peptide. Importantly, in $\mathrm{MOG}_{35-55}$ immunized calnexin-null mice without neurobehavioral deficits, circulating lymphocytes secreted proinflammatory cytokines but showed no signs of immune cell infiltration into the CNS or induction of transcripts encoding inflammatory genes within the CNS. The importance of calnexin in the susceptibility to inflammatory demyelination was demonstrated by transgene-directed reexpression of calnexin in $\mathrm{Can}^{-1-}$ mice. The seemingly paradoxical results of this study, demonstrating that calnexin deficiency leads to dysmyelination and impaired peripheral motor function (18-20) while conferring protection against inflammation of the CNS in response to induction of EAE, suggest that calnexin is important for myelin formation, but in EAE, an elevated abundance of calnexin is associated with the pathogenic cascade that eventually culminates in the progressive destruction of the myelin. 


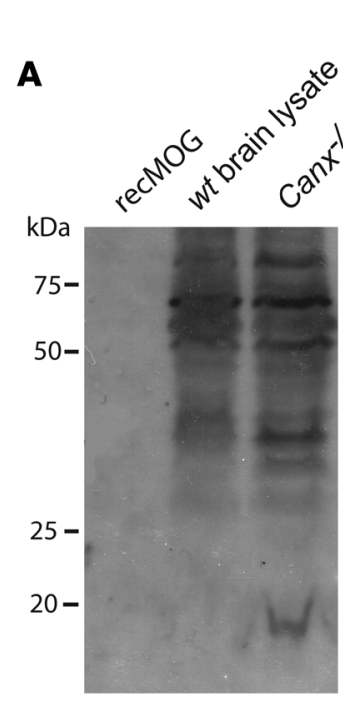

IB: serum
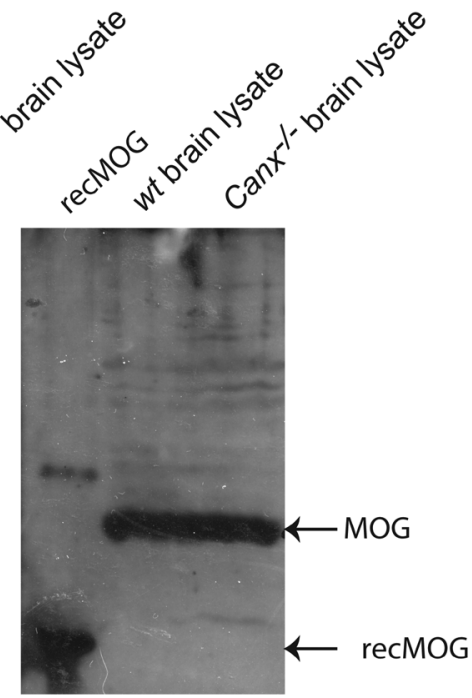

from wild-type mice from $\mathrm{MOG}_{35-55}$

injected wild-type mice

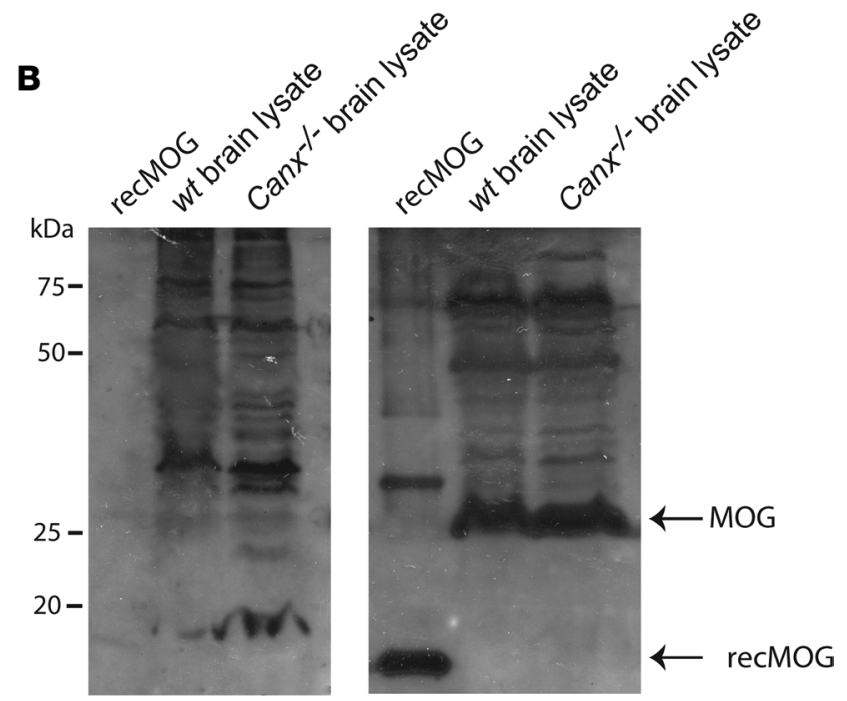

IB: serum from $\mathrm{Canx}^{-/-}$mice
IB: serum

from $\mathrm{MOG}_{35-55}$ injected $\mathrm{Canx}^{-} \%-$ mice

C

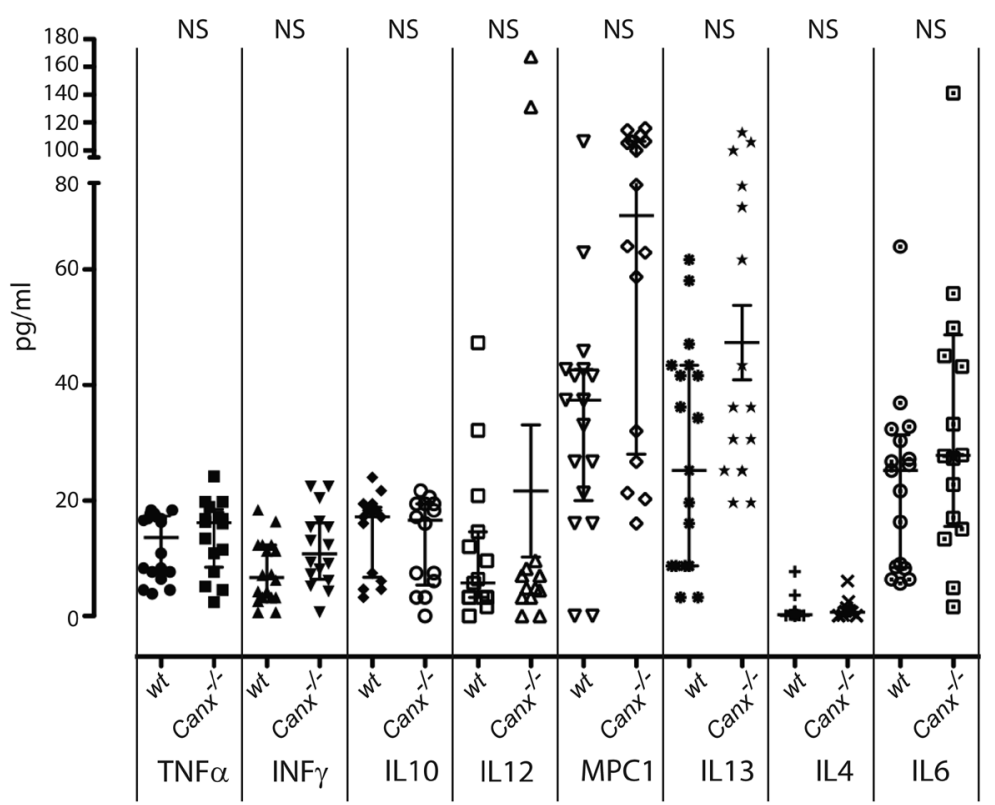

D

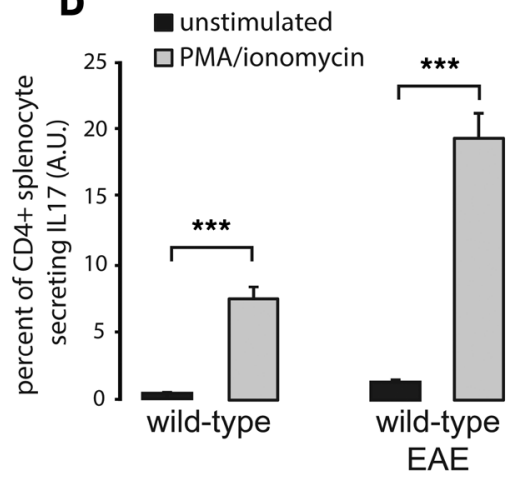

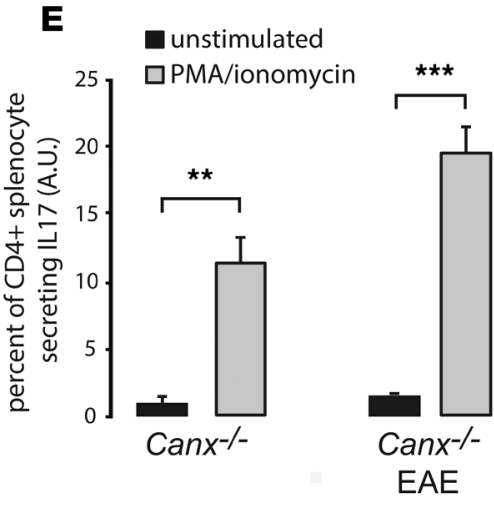

Figure 5. Peripheral immune response in WT and calnexin-deficient EAE mice. (A) Immunoblot (IB) analysis of brain extracts from WT or calnexin-deficient mice probed with serum collected from WT mice (left panel) or WT mice immunized with $\mathrm{MOC}_{35-55}$ peptide (right panel). The location of the recombinant MOC (recMOG) and the endogenous brain MOC is indicated by the arrows $(n=3)$. (B) Immunoblot (IB) analysis of brain extracts from WT or Canx ${ }^{-/-}$mice probed with serum collected from Canx $x^{-/-}$mice (left panel) or Canx ${ }^{-/-}$immunized with $\mathrm{MOG}_{35-55}$ peptide (right panel). The location of the recMOG and the brain MOG is indicated by the arrows. (C) Selected cytokine production in the periphery in WT and Canx ${ }^{-1-}$ mice. Data presented are mean \pm SEM of 5 independent experiments (15-19 mice per experimental group). Statistical analysis by unpaired 2-tailed Student's $t$ test. (D) IL-17 secretion by $\mathrm{CD}^{+}$splenocytes isolated from WT or EAE WT mice. Cells were stimulated with PMA/ionomycin. All data are representative of 3 biological replicates. (E) Percentage of activated $\mathrm{CD}^{+}$splenocytes from $\mathrm{Canx}^{-/-}$mice of or EAE Canx $x^{-/-}$mice secreting IL-17. ${ }^{* *} P \leq$ $0.01,{ }^{* *} P \leq 0.001 ; n=3$. The means were compared using unpaired 2-tailed Student's $t$ test. See complete unedited blots in the supplemental material. 
Table 1. Clinical features of $\mathrm{MOG}_{35-55}$-induced EAE in Canx ${ }^{f l / f l} / \mathrm{Lck}_{\mathrm{S}}-\mathrm{Cre}^{+}$mice with calnexindeficient $T$ cells.

\begin{tabular}{lcc}
\hline Genotype & Mean day of disease onset & Mean max score \\
WT mice & $10.2 \pm 0.5$ & $3.8 \pm 0.2$ \\
Canx $x^{-/-}$mice & No symptoms & No symptoms \\
Canx $^{f / f l}$ mice & $10 \pm 0.42$ & $3.5 \pm 0.3$ \\
Lck-Cre $^{+}$mice & $8.75 \pm 0.35$ & $3.37 \pm 0.55$ \\
Canx $^{f l / f l} /$ Lck-Cre mice & $7.5 \pm 1.7$ & $3.8 \pm 0.35$ \\
Data shown are mean \pm SEM; $n=4$. & & \\
\hline
\end{tabular}

MS is classified as a chronic progressive autoimmune disease of the CNS in which proinflammatory $\mathrm{T}$ cells, sensitized to myelin proteins, cross the blood-brain barrier, resulting in inflammatory demyelination. EAE in mice, an established model of MS, is characterized by extensive infiltration of the CNS by inflammatory cells (22). Initiation of EAE involves the activation of myelin-specific Th17 cells (22). Th17 cells, which produce inflammatory cytokines, including IL-17,

are critical mediators of tissue damage in EAE (22). We found no difference in IL-17 secretion between $\mathrm{T}$ cells isolated from WT and $\mathrm{Canx}^{-/-}$mice immunized with $\mathrm{MOG}_{35-55}$, ruling out any contribution of IL-17 in EAE protection seen in the calnexin-deficient mice. Importantly, mice with targeted inactivation of the calnexin gene in $\mathrm{T}$ cells offered no resistance to EAE, as these mice developed EAE to the same level of severity exhibited by WT mice with EAE. These findings indicated that the absence of calnexin did not affect activation and expansion of myelin-specific $\mathrm{T}$ cells in the periphery during EAE, despite the relative protection against EAE development in calnexin-deficient mice.

Since $\mathrm{T}$ cell-specific inactivation of calnexin did not recapitulate the resistance to EAE exhibited by mice with global calnexin deficiency, we examined the transmigration of $\mathrm{T}$ cells across the blood-brain barrier using bEND. 3 cells as a model of brain endothelial cells (29). Canx ${ }^{/-}$-bEND. 3 cells showed normal responses to stimulation by cytokines with respect to upregulation of cell adhesion molecules but failed to support transendothelial migration of $\mathrm{T}$ cells. This finding demonstrates that blocking of $\mathrm{T}$ cell migration across the blood-brain barrier underlies the observed resistance of calnexin-deficient mice to EAE. Blockage of lymphocytes binding to brain endothelium and reduction of lymphocyte transendothelial migration into brain parenchyma has long been considered a target for MS therapeutics development $(31,32)$. Several adhesion molecules have been implicated in the binding of lymphocytes to inflamed blood vessels in the brain of mice with EAE. Blockade of VCAM1 interaction with integrin $\alpha 4$ localized on the cell surface of lymphocytes reduces the clinical severity of EAE (32) and is the basis for MS therapy. Interestingly, antibodies inhibiting binding of leukocytes to other endothelial proteins, including ICAM1, do not influence lymphocyte binding to the blood-brain barrier (33).

Cytokine-stimulated Canx $x^{-1-}$-bEND. 3 cells express increased levels of VCAM1, ICAM1, and tight junction proteins, yet transendothelial migration of $\mathrm{T}$ cells is effectively blocked to protect calnexin-deficient mice against EAE. Calnexin is a type 1 integral ER membrane protein with a large portion of the C-terminal domain oriented to the cytoplasm $(34,35)$ and interacting with cytoplasmic proteins, including UBC9 (SUMOylation E2 ligase) (36), protein tyrosine phosphatase 1B (36), N-myristoyltransferase 1 (37), and SGIP1 (16). While screening for calnexin interacting partner proteins in the nervous system, we discovered that the fatty acid binding protein 5 (Fabp5), a member of a family of highly conserved cytoplasmic proteins that bind long-chain fatty acids and other hydrophobic ligands, binds to the cytoplasmic domain of calnexin (38). It has been reported that a deficiency in Fabp5 also protects against EAE $(39,40)$. It is tempting to speculate that the calnexin/Fabp5 complex is a component of neuroproteostasis and lipidostasis (41). The dysregulation of calnexin/Fabp5 function may be a key event in the pathogenesis of MS and represents an important target for therapeutics development aimed at promoting favorable clinical outcomes for patients afflicted with MS.

\section{Methods}

Human CNS tissue and grading. Snap-frozen postmortem CNS tissue blocks were obtained from both normal $(n=10)$ and MS $(n=18)$ cases with varying degrees of lesion inflammation at autopsy, with donor or next-of-kin informed consent for tissue donation from the NeuroResource tissue bank. MS lesions were classified into acute, subacute, and chronic on the basis of the number and distribution of Oil Red O-positive macrophages, myelin loss, and the cellularity in the borders and parenchyma of lesions. Clinical data were collected as shown in Supplemental Table 1 (42).

Mouse models. Calnexin-deficient mice were generated as previously described (18). To inactivate the 
A

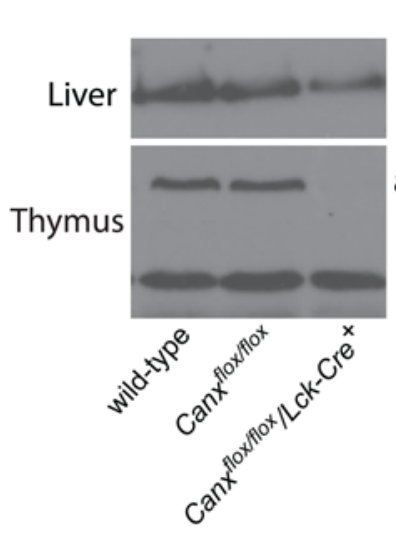

B

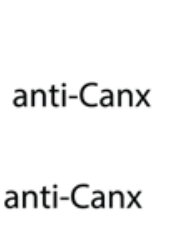
anti-GAPDH
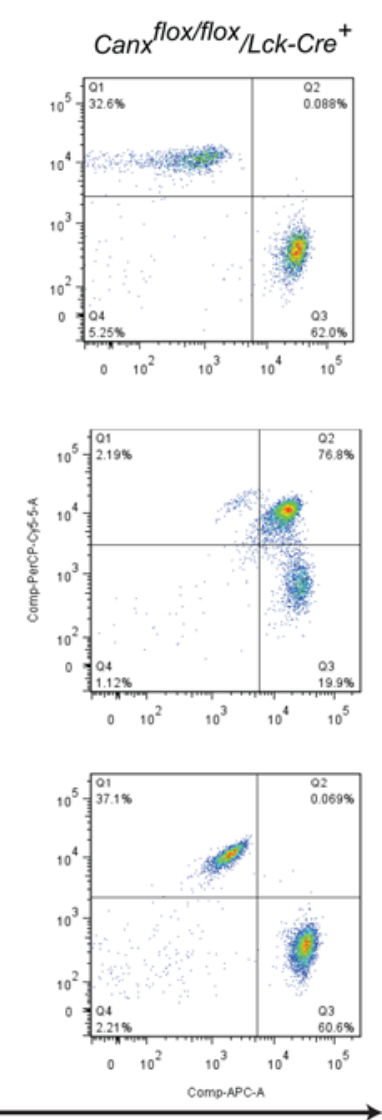

Figure 6. Targeted inactivation of the calnexin gene in T cells. (A) Immunoblot analysis of liver and thymus extracts from WT,

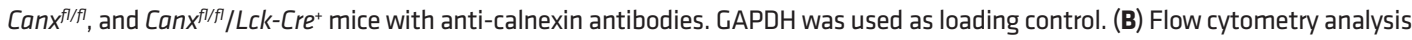
of the peripheral immune system in WT and $C a n x^{f / f / f} / L c k-C r e^{+}$mice. Thymocytes, splenocytes, and lymphatic node cells were analyzed for expression of $\mathrm{CD}^{+} \mathrm{CD} 8^{+}$antigens. The $\mathrm{CD} 4^{+} \mathrm{CD} 88^{+}$profiles of peripheral immune cells are not changed in transgenic animals. Data are representative of 3 biological replicates. See complete unedited blots in the supplemental material.

calnexin gene in the T cell lineage, mice carrying floxed calnexin exons (exon 3 and 4) Canx $x^{A / f}(27)$, were crossed with $\mathrm{Lck}_{\mathrm{C}} \mathrm{Cre}^{+}$transgenic mice (C57BL/6), which express the Cre recombinase under the control of the T cell-specific Lck promoter (The Jackson Laboratory, B6.Cg-Tg[Lck-cre]548Jxm/J]. Presence of the flox and Cre recombinase alleles was confirmed by PCR analysis using the following set of DNA primers: 5'-GCTCTGGATGCCTTGGGATTTGATTTCC-3'; 5'-GGATTTAGTGGATATCCCCAGTATGAGC-3'; Cre-F2, 5'-GCCAGCTAAACATGCTTCATC-3'; Cre-B2, 5'-ATTGCCCCTGTTTCACTATCC-3'. Mice used in the present study carried double-flox and single Cre recombinase alleles.

An expression vector designed for ubiquitous expression under the ROSA26 promoter was used to generate transgenic mice expressing full-length calnexin protein on a CD1 WT mouse background, designated Canx $x^{-1}$-TG-CanxFL. Transgene expression was determined by PCR using the following set of DNA primers: $2 \mathrm{~N}$ forward, 5'-GGAGGAGCGCTTCCGGCCGACG-3'; 2N reverse, 5'-CCACCCTGACAGAGACCCTCTGTC-3'; 2C forward, 5'-CGGAGAATGGGAGGCTCCTCAGAT T-3'; 2C reverse, 5'-GCAAGAAAGCGAGCTTCTAGATGGCC-3'. Mice were bred by crossing CD1 WT mice expressing full-length calnexin transgene with WT C57BL/6 mice. To generate calnexin rescue mice, WT CD1/ C57BL6 mice expressing the transgene were crossed with calnexin-deficient mice, and the heterozygote progeny with the transgene were bred again with calnexin-deficient mice, producing animals with the transgene on a calnexin-deficient background. Multifiber motor and sensory conduction studies were carried out using previously published protocols (18).

EAE induction. Adult mice were injected s.c. in each hind leg with $50 \mu \mathrm{g}$ of $\mathrm{MOG}_{35-55}$ (Peptide Synthesis Facility, University of Alberta) emulsified in $100 \mu \mathrm{l}$ CFA (MilliporeSigma). Animals received 0.3 $\mu \mathrm{g} / \mathrm{ml}$ of pertussis toxin injected i.p. Injection of pertussis toxin (List Biological Laboratories) occurred 
A

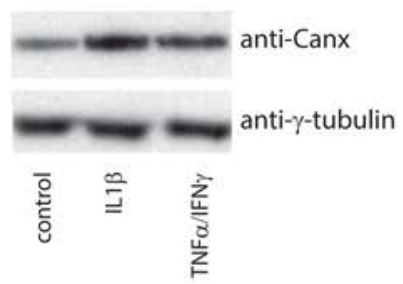

B

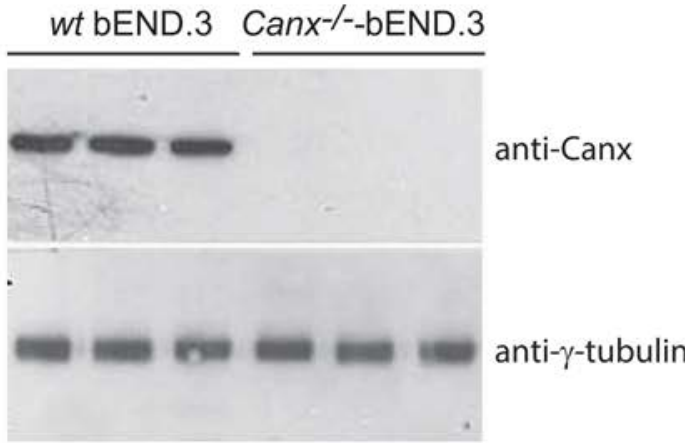

C

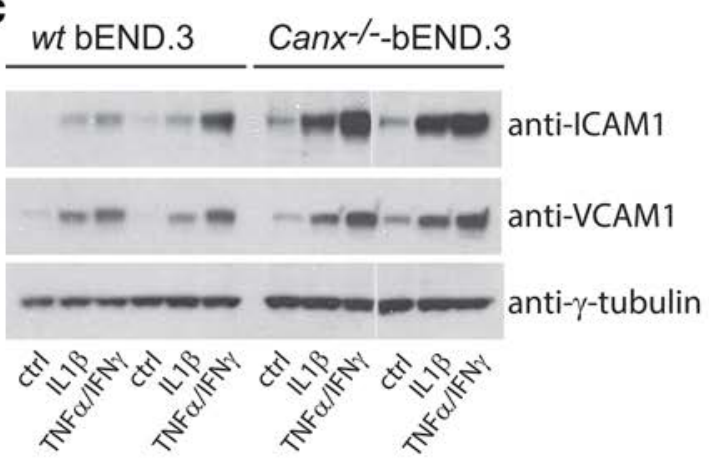

D

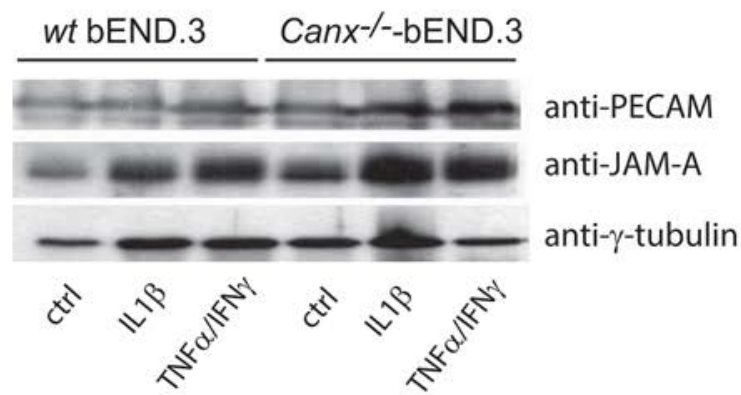

$\mathbf{E}$
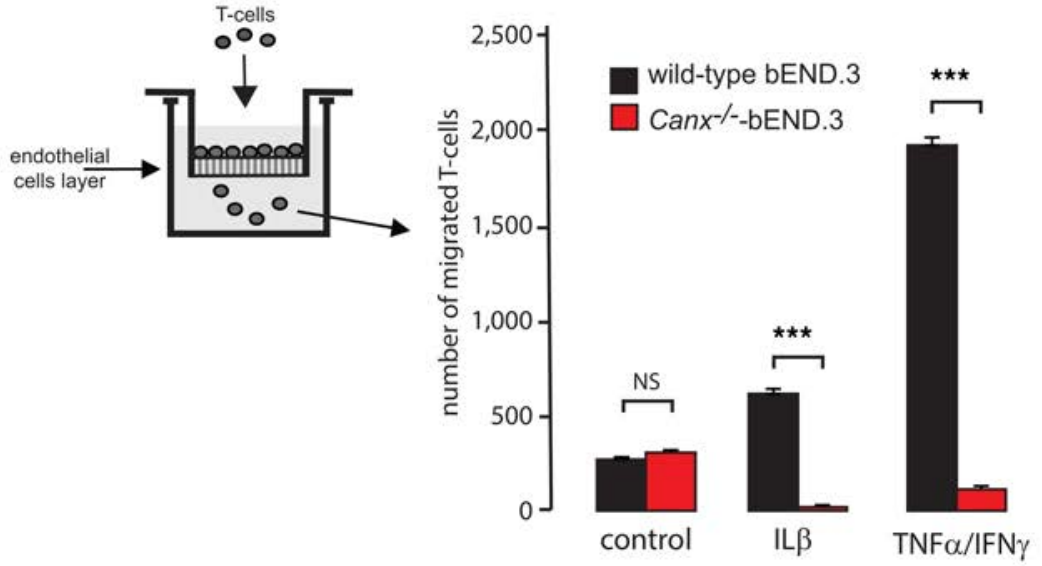

Figure 7. Blood-brain barrier endothelial cell analysis. (A) Calnexin protein expression in stimulated (with IL-1 $\beta$ or TNF- $\alpha /$ IFN- $\gamma$ ) bEND. 3 cells. Immunoblot analysis of bEND. 3 cells with anti-calnexin antibodies. $\gamma$-Tubulin was used as loading control. (B) Immunoblot analysis of bEND.3 cells with the CRISPR/Cas9 silenced calnexin gene. Immunoblot analysis was carried out with anti-calnexin antibodies raised against a peptide derived from the C-terminus of calnexin. $\gamma$-Tubulin was used as the loading control. (C) Abundance of VCAM and ICAM in WT and Canx ${ }^{-/-}$-bEND. 3 cells stimulated with IL-1 $1 \beta$ or TNF- $\alpha /$ IFN- $\gamma \cdot \gamma$-Tubulin was used as loading control. Anti-ICAM1 and anti- $\gamma$-tubulin lanes were run on the same gel but were noncontiguous, as indicated by a thin white vertical line. (D) Abundance of PECAM and JAM-A in WT and Canx $x^{-/-}$-bEND. 3 cells stimulated with IL-1 $\beta$ or TNF- $\alpha /$ /FN- $\gamma$. $\gamma$-Tubulin was used as loading control. (E) Quantification of activated WT splenocytes that had migrated across WT or Canx ${ }^{-/-}$-bEnd.3 cells. ${ }^{* * *} P \leq$ 0.001 . Data presented are mean \pm SEM of 3 independent transmigration experiments with 3 replicates. The means were compared using unpaired 2-tailed Student's $t$ test. See complete unedited blots in the supplemental material.

on the day of induction with $\mathrm{MOG}_{35-55}$, with subsequent infection 48 hours later. Control animals were immunized with CFA and PBS (1:1) and pertussis toxin, as above. Animals were assessed daily for EAE clinical symptoms. A scoring system from $0-5$ for clinical assessment of EAE symptoms was used (https://hookelabs.com/services/cro/eae/MouseEAEscoring.html). EAE scores were obtained daily and were calculated for each mouse during the time course of the disease. Time course graphs represent daily mean maximal scores. Mean EAE data is shown as \pm SEM.

Measurement of anti-MOG antibodies and cytokines in serum. Sera of control and EAE mice were collected 
17 days after immunization. Levels of serum cytokines (GM-CSF, INF- $\gamma$, IL-1 $\beta$, IL-2, IL-4, IL-5, IL-6, IL-7, IL-10, IL-12, IL-13, MCP-1, TNF- $\alpha$ ) were evaluated using the Milliplex MAP Mouse Cytokine/ Chemokine Immunoassay (MilliporeSigma, Milliplex MAP Kit Mouse Cytokine/Chemokine 96-well plate assays, MPXMCYTO70KPMX13) according to the manufacturer's instructions.

Histochemistry. Mice were perfused with PBS, with the brain and spinal cord tissues fixed in $10 \%$ formalin and with paraffin-embedded sections stained with H\&E. IHC staining of formalin-fixed, paraffin embedded lumbar section of spinal cords were deparaffinised and rehydrated using decreasing concentrations of ethanol. The slides were boiled in $0.01 \mathrm{M}$ trisodium citrate buffer, $\mathrm{pH} 6$, for 20 minutes followed by incubation with $0.3 \%$ hydrogen peroxide to block endogenous peroxidases. Sections were then preincubated with blocking buffer (LI-COR) for several hours to block nonspecific binding. To detect selected immune cells markers, sections were incubated overnight at $4^{\circ} \mathrm{C}$ with anti-CD3 $\varepsilon$ (Serotec, catalog MCA500GA), anti-F4/80 (Abcam, catalog AB6640), anti-IBA1 (Wako Pure Chemical Industries Ltd., catalog 019-19741), anti-GFAP (Dako, catalog M0761) antibodies at 1:100 dilution, followed by secondary biotinylated antibody, avidin-biotin-peroxidase complexes (Vector Laboratories) and 3,3'-diaminobenzidine tetrachloride (Vector Laboratories). For quantification, spinal cord sections were scanned and photographed using Axioskop2 (Carl Zeiss MicroImaging Inc.). For quantitative analysis, a number of cells positive for CD3e, IBA-1, F4/80, and GFAP were determined in 3 standardized, randomly placed fields of view of $50 \mu \mathrm{m}^{2}$. Cells were counted within the white matter around the dorsal horn of the spinal segments using Optical Light Microscope (Optica).

Cell culture and generation of the Canx $x^{-1-}-b E N D .3$ cell line. The bEND.3 cell line was purchased from ATCC (CRL-2299) and cultured under recommended conditions. Cells were cultured in DMEM media supplemented with $10 \%$ (vol/vol) FBS at $37^{\circ} \mathrm{C}$ and $5 \% \mathrm{CO}_{2}$. Disruption of the calnexin gene in the bEND.3 cell line was achieved with 2 paired sgRNAs designed to excise a large part of the gene. We used sgRNAs $5^{\prime}$ CACCGTAGTATAGGGAGTCTTGTCG - 3'; 5' - CACCGTAAACCTCCCCTGTTGGAAC - 3', which were generated with the web-based tool http://crospr/mit.edu. Construction of the sgRNA coding vector was performed by ligating each sgRNA into the plasmid SpCas9(BB)-2A-Puro (Px459) V2.0 (a gift from Feng Zhang [Broad Institute of Massachusetts Institute of Technology, Cambridge, Massachusetts, USA; Addgene plasmid 62988]). Top and bottom oligos were prepared and ligated with the vector as described (43). DH5 $\alpha$ E. coli-competent cells were transformed with the ligation mixture. Colonies were picked, and DNA extraction was performed. Positive ligation was confirmed with DNA sequence analysis. bEND.3 cells at a confluency of 70\%-90\% were transfected using TurboFect Transfection reagent (Thermo Fisher Scientific). Transfection was performed according to manufacturer's recommended protocol designed for adherent cells. Pairs of sgRNAs were used to mediate effective gene disruption. Individual clones were isolated from the cell population transfected with sgRNAs and assayed by both PCR and immunoblot analysis, to confirm successful inactivation of the calnexin gene. For PCR analysis, genomic DNA from the modified region was amplified using primers outside of the sgRNAs binding sites: forward, 5'-GCCTTGGGATTTGATTTCCT-3'; reverse, 5'-GATCTGCATCTGGCCT-3'. PCR product amplified from transfected bEND. 3 cells reflected a deletion of $3,198 \mathrm{bp}$. To further confirm deletion of part of the calnexin gene, the amplicon was sequenced to reveal the exact sites of the deletion (Supplemental Figure 2). Immunoblot analysis was carried out with anti-calnexin antibodies raised against a peptide derived from the C-terminal of calnexin (Assay Designs, SPA-860, synthetic peptide corresponding to last 19 C-terminal residues of amino acid sequence of calnexin [residues 575-593; $\mathrm{NH}_{3}{ }^{+}$-AEEDEI LNRSPRNRKP RRE-COO-]).

Transendothelial migration analysis. WT or calnexin-deficient bEND.3 cells were plated onto 12-well plates and cultured for 24-48 hours until cells formed an intact monolayer. The endothelial cell monolayer was treated for 24 hours with IL-1 $\beta$ (10 ng/ml) (eBioscience, Affymetrix) or TNF- $\alpha$ ( $3 \mathrm{ng} / \mathrm{ml})$ (Invitrogen), and IFN- $\gamma(60 \mathrm{ng} / \mathrm{ml})$ (eBioscience, Affymetrix) added to the culture media. For transmigration experiments, bEND. 3 cells were plated onto transwell inserts (Corning, $5 \mu \mathrm{m}$ ). Primary cultures of WT mouse splenocytes were isolated. Briefly, spleens were carefully removed, sliced into small pieces and placed on a $70-\mu \mathrm{m}$ cell strainer, and treated with RBC lysing buffer according to the protocol, with a cell suspension of $1 \times 10^{6} \mathrm{cells} / \mathrm{ml}$ in the final preparation. Cells were activated with plate-bound CD3 antibodies $(5 \mu \mathrm{g} /$ $\mathrm{ml}$ eBioscience) and soluble CD28 antibodies ( $2 \mu \mathrm{g} / \mathrm{ml}$ eBioscience). On the day of the experiment, $\mathrm{T}$ cells were labeled with CFSE (Invitrogen) according to manufacturer's protocol. The CFSE-labeled cell suspension was added to the monolayer of bEND.3 cells and incubated for 3 hours. Next, fluorescently labeled leucocytes from the lower well were collected, washed 3 times with PBS, and counted using flow cytometry.

Flow cytometry. Flow cytometry analysis was carried out using BD FACSCanto flow cytometer (BD 
Biosciences) and FACSDiva software (BD Biosciences) for acquisition and FlowJo for analysis (Tree Star Inc.). Cells were washed in PBS with $2 \%$ heat-inactivated FBS and stained with designated cocktails of fluorochrome-conjugated antibodies for 1 hour at $4^{\circ} \mathrm{C}$ in the dark. Fluorochrome conjugated monoclonal antibodies were obtained from eBioscience and included: CD3e (clone 145-2C11), CD4 (clone RM4-5), CD8a (clone 53-67), CD11b (clone M1/70), CD11c (clone N418), CD69 (clone H1.2F3), MHC I (clone $\mathrm{H}-2 \mathrm{Db}), \mathrm{MHC}$ II (clone I-A/I-E).

Quantitative PCR. Murine lumbar spinal cords were homogenized in TRIzol reagent (Invitrogen), and total RNA was isolated and purified using RNeasy mini columns (Qiagen). Complementary DNA (cDNA) was prepared by oligo(dT)-primed reverse transcription of mRNA using iScript cDNA Synthesis Kit (Bio-Rad) according to the manufacturer's recommended protocols. Quantitative PCR (qPCR) was performed using Bio-Rad iQ 2 SYBR green supermix, using the Rotor Gene Q (Qiagen), according to the manufacturer's recommended protocols.

T cell activation and cell proliferation assays. Purified CD4 ${ }^{+} \mathrm{T}$ cells from healthy control WT mice were cultured in 24-well plates $\left(1 \times 10^{6} / \mathrm{ml}\right)$ and stimulated with anti-CD3 and anti-CD28 antibodies (eBioscience; $5 \mu \mathrm{g} / \mathrm{ml}$ each). Culture plates were then incubated for 48 hours at $37^{\circ} \mathrm{C}$.

Splenocytes were isolated from EAE-induced C57BL/6 mice at day 17. Briefly, total splenocytes were seeded at $2 \times 10^{5}$ cells/well in a 96-well round-bottomed plate with or without $\mathrm{MOG}_{35-55}(20 \mu \mathrm{g} / \mathrm{ml}) \mathrm{pep}$ tide for 5 days, followed by subsequent fixation and flow cytometric analysis. DMEM supplemented with 10\% FBS (Invitrogen), 1\% penicillin/streptomycin (Invitrogen), $2 \mathrm{mM} \mathrm{L-glutamine} \mathrm{(Invitrogen),} \mathrm{and} \mathrm{1 \%}$ nonessential amino acids (Invitrogen) were used for cell culture.

IL-17 secretion assay. Mouse IL-17 Secretion Assay Cell Enrichment and Detection Kit (MACS, Miltenyi Biotec) was performed according to the manufacturer's protocol. Mouse splenocytes were stimulated with PMA/ionomycin for 4 hours to achieve IL-17 secretion. Subsequently, cells were incubated with IL-17-specific Catch reagent that attached to the cell surface. Secreted IL-17 was bound to the Catch reagent; next, positive cells were labeled with IL-17-specific antibody. To detect IL-17-secreting positive cells, mouse IL-17 detection antibody conjugated with biotin and anti-biotin-R-phycoerythrin (PE) antibodies were used. Cells were magnetically labeled with anti-PE microbeads that allowed enrichment of the IL-17-secreting cells over a MACS column placed in the magnetic field of the MACS separator. Magnetically labeled cells bound to the column and were later eluted and analyzed by flow cytometry.

Statistics. Statistical analysis was performed using GraphPad Software, and results are presented as the mean \pm SEM. Two-tailed Student's $t$ tests were performed when appropriate. A significant difference was determined as follows: ${ }^{*} P \leq 0.05 ; * * P \leq 0.01 ; * * * P \leq 0.001$.

Study approval. All methods were carried out in accordance with relevant guidelines and regulations and were approved by Biosafety Officers at the Department of Environment, Health and Safety at the University of Alberta. All animal experiments were carried out according to the University of Alberta Animal Policy and Welfare Committee and the Canadian Council on Animal Care Guidelines. The approval for use of animals in research was granted by the ACUC for Health Sciences, a University of Alberta ethics review committee (permit AUP297). All animal experimentation was carried out working closely with University of Alberta animal facility staff and veterinarian. Donor or next-of-kin informed consent for tissue donation was obtained from the NeuroResource tissue bank (UCL Institute of Neurology, London, United Kingdom [ethical approval ID. 08/H0718/54+5 from NRES Committee London - Central] or from the Multiple Sclerosis Society Tissue Bank [ethical approval reference number 08/MRE09/31], and approval for our study was obtained from the Local Research Ethics Committee [ID 04/Q2102/111]).

\section{Author contributions}

JJ designed experiments; analyzed data; performed biochemical, biophysical, cell biological, and animal experiments; and wrote the manuscript. PE designed experiments, analyzed data, performed human sample analyses, and wrote the manuscript. NG, JH, and JN designed experiments, analyzed data, and performed human sample analyses. AR carried out animal experimentation. ED and JW designed experiments and performed cell biological analysis. AMP and CP designed experiments, analyzed data, and assisted in EAE experimentation. DZ designed experiments, analyzed data, and performed electrophysiological studies. AK designed experiments, analyzed data, and performed biochemical and animal analyses. LBA and MM designed experiments, analyzed data, and wrote the manuscript. 


\section{Acknowledgments}

We thank Britta Engelhardt (University of Bern, Bern, Switzerland) for many helpful discussions during the course of this work. We thank Jody Groenendyk for critical reading of the manuscript and helpful discussions. We thank Bhagat Singh for assistance with electrophysiological studies and William Branton for help with histological analysis. Tissue samples and associated clinical and neuropathological data were supplied by NeuroResource, UCL Institute of Neurology, United Kingdom, and also the Multiple Sclerosis Society Tissue Bank, funded by the Multiple Sclerosis Society of Great Britain and Northern Ireland, registered charity 207495. This work was supported by the Canadian Institutes of Health Research grants MOP-15291, MOP-15415, and PS-153325 to MM and MOP-15291, MOP-86750, and PS-153325 to LBA; by a generous donation from Kenneth McCourt family; and by the Multiple Sclerosis Society of Canada (EGID2426) to CP. Additional support was provided by research grants from the Royal Devon and Exeter Hospital Foundation Trust and Northcott Devon Medical Foundation to PE, JEH, and NJG and a generous donation from Sheryl Moorey's family. JJ and AK were supported by the Alberta Innovates-Health Solutions (AI-HS) studentship. AK was also supported by a Multiple Sclerosis Society of Canada studentship. ED was supported by the AI-HS postdoctoral fellowship. JW was supported by the Alberta MS Network summer studentship award.

Address correspondence to: Marek Michalak, 3-55 Medical Sciences Building, Department of Biochemistry, University of Alberta, Edmonton, Alberta, Canada T6G 2H7. Phone: 780.492.2256; Email: marek.michalak@ualberta.ca. Or to: Luis B. Agellon, School of Dietetics and Human Nutrition, McGill University, Ste. Anne de Bellevue, Quebec, Canada H9X 3V9. Phone: 514-398-7862. Email: luis.agellon@mcgill.ca.

AK's present address is: Rocky Mountain Laboratories, National Institute of Allergy and Infectious Disease, National Institutes of Health, Hamilton, Montana, USA.

1. Kingwell E, et al. Incidence and prevalence of multiple sclerosis in Europe: a systematic review. BMC Neurol. 2013;13:128.

2. Kingwell E, et al. High incidence and increasing prevalence of multiple sclerosis in British Columbia, Canada: findings from over two decades (1991-2010). J Neurol. 2015;262(10):2352-2363.

3. Frohman EM, Racke MK, Raine CS. Multiple sclerosis--the plaque and its pathogenesis. N Engl J Med. 2006;354(9):942-955.

4. Tietz S, Engelhardt B. Brain barriers: Crosstalk between complex tight junctions and adherens junctions. J Cell Biol. 2015;209(4):493-506.

5. Banks WA. From blood-brain barrier to blood-brain interface: new opportunities for CNS drug delivery. Nat Rev Drug Discov. 2016;15(4):275-292

6. Zlokovic BV. The blood-brain barrier in health and chronic neurodegenerative disorders. Neuron. 2008;57(2):178-201.

7. Steiner O, et al. Differential roles for endothelial ICAM-1, ICAM-2, and VCAM-1 in shear-resistant T cell arrest, polarization, and directed crawling on blood-brain barrier endothelium. J Immunol. 2010;185(8):4846-4855.

8. Privratsky JR, Newman PJ. PECAM-1: regulator of endothelial junctional integrity. Cell Tissue Res. 2014;355(3):607-619.

9. McCaffrey K, Braakman I. Protein quality control at the endoplasmic reticulum. Essays Biochem. 2016;60(2):227-235

10. Hebert DN, Molinari M. In and out of the ER: protein folding, quality control, degradation, and related human diseases. Physiol Rev. 2007;87(4):1377-1408.

11. Krebs J, Agellon LB, Michalak M. Ca(2+) homeostasis and endoplasmic reticulum (ER) stress: An integrated view of calcium signaling. Biochem Biophys Res Commun. 2015;460(1):114-121.

12. Coe H, Michalak M. Calcium binding chaperones of the endoplasmic reticulum. Gen Physiol Biophys. 2009;28 Spec No Focus:F96-F103

13. Parlati F, Dignard D, Bergeron JJ, Thomas DY. The calnexin homologue cnx1+ in Schizosaccharomyces pombe, is an essential gene which can be complemented by its soluble ER domain. EMBO J. 1995;14(13):3064-3072.

14. Lee W, et al. Alternative chaperone machinery may compensate for calreticulin/calnexin deficiency in Caenorhabditis elegans. Proteomics. 2006;6(4):1329-1339.

15. Müller-Taubenberger A, Lupas AN, Li H, Ecke M, Simmeth E, Gerisch G. Calreticulin and calnexin in the endoplasmic reticulum are important for phagocytosis. EMBO J. 2001;20(23):6772-6782.

16. Li HD, Liu WX, Michalak M. Enhanced clathrin-dependent endocytosis in the absence of calnexin. PLoS One. 2011;6(7):e21678

17. Coe H, Bedard K, Groenendyk J, Jung J, Michalak M. Endoplasmic reticulum stress in the absence of calnexin. Cell Stress Chaperones. 2008;13(4):497-507.

18. Kraus A, et al. Calnexin deficiency leads to dysmyelination. J Biol Chem. 2010;285(24):18928-18938.

19. Jung J, Coe H, Michalak M. Specialization of endoplasmic reticulum chaperones for the folding and function of myelin glycoproteins P0 and PMP22. FASEB J. 2011;25(11):3929-3937.

20. Denzel A, et al. Early postnatal death and motor disorders in mice congenitally deficient in calnexin expression. Mol Cell Biol. 2002;22(21):7398-7404.

21. Peschl P, Bradl M, Höftberger R, Berger T, Reindl M. Myelin Oligodendrocyte Glycoprotein: Deciphering a Target in Inflammatory Demyelinating Diseases. Front Immunol. 2017;8:529. 
22. Constantinescu CS, Farooqi N, O'Brien K, Gran B. Experimental autoimmune encephalomyelitis (EAE) as a model for multiple sclerosis (MS). Br J Pharmacol. 2011;164(4):1079-1106.

23. Komiyama Y, et al. IL-17 plays an important role in the development of experimental autoimmune encephalomyelitis. $J$ Immunol. 2006;177(1):566-573.

24. Shimizu C, et al. Progression of T cell lineage restriction in the earliest subpopulation of murine adult thymus visualized by the expression of lck proximal promoter activity. Int Immunol. 2001;13(1):105-117.

25. Chiang YJ, Hodes RJ. T-cell development is regulated by the coordinated function of proximal and distal Lck promoters active at different developmental stages. Eur J Immunol. 2016;46(10):2401-2408.

26. Zhang DJ, et al. Selective expression of the Cre recombinase in late-stage thymocytes using the distal promoter of the Lck gene. J Immunol. 2005;174(11):6725-6731.

27. Tokuhiro K, et al. Calreticulin is required for development of the cumulus oocyte complex and female fertility. Sci Rep. 2015;5:14254

28. Sikorski EE, Hallmann R, Berg EL, Butcher EC. The Peyer's patch high endothelial receptor for lymphocytes, the mucosal vascular addressin, is induced on a murine endothelial cell line by tumor necrosis factor-alpha and IL-1. J Immunol. 1993;151(10):5239-5250.

29. Watanabe T, et al. Paracellular barrier and tight junction protein expression in the immortalized brain endothelial cell lines bEND.3, bEND.5 and mouse brain endothelial cell 4. Biol Pharm Bull. 2013;36(3):492-495.

30. Wearsch PA, Cresswell P. The quality control of MHC class I peptide loading. Curr Opin Cell Biol. 2008;20(6):624-631

31. Shlosberg D, Benifla M, Kaufer D, Friedman A. Blood-brain barrier breakdown as a therapeutic target in traumatic brain injury. Nat Rev Neurol. 2010;6(7):393-403

32. Miller DH, et al. A controlled trial of natalizumab for relapsing multiple sclerosis. N Engl J Med. 2003;348(1):15-23

33. Steinman L. Blocking adhesion molecules as therapy for multiple sclerosis: natalizumab. Nat Rev Drug Discov. 2005;4(6):510-518.

34. Ho SC, Rajagopalan S, Chaudhuri S, Shieh CC, Brenner MB, Pillai S. Membrane anchoring of calnexin facilitates its interaction with its targets. Mol Immunol. 1999;36(1):1-12.

35. Chevet E, Smirle J, Cameron PH, Thomas DY, Bergeron JJ. Calnexin phosphorylation: linking cytoplasmic signalling to endoplasmic reticulum lumenal functions. Semin Cell Dev Biol. 2010;21(5):486-490.

36. Lee D, et al. UBC9-dependent association between calnexin and protein tyrosine phosphatase $1 \mathrm{~B}$ (PTP1B) at the endoplasmic reticulum. J Biol Chem. 2015;290(9):5725-5738.

37. Dudek E, Millott R, Liu WX, Beauchamp E, Berthiaume LG, Michalak M. N-Myristoyltransferase 1 interacts with calnexin at the endoplasmic reticulum. Biochem Biophys Res Commun. 2015;468(4):889-893.

38. Jung J, Wang J, Groenendyk J, Lee D, Michalak M, Agellon LB. Fatty acid binding protein (Fabp) 5 interacts with the calnexin cytoplasmic domain at the endoplasmic reticulum. Biochem Biophys Res Commun. 2017;493(1):202-206.

39. Rao E, et al. Targeting epidermal fatty acid binding protein for treatment of experimental autoimmune encephalomyelitis. $B M C$ Immunol. 2015;16:28.

40. Li B, Reynolds JM, Stout RD, Bernlohr DA, Suttles J. Regulation of Th17 differentiation by epidermal fatty acid-binding protein. J Immunol. 2009;182(12):7625-7633.

41. Jung J, Michalak M, Agellon LB. Endoplasmic Reticulum Malfunction in the Nervous System. Front Neurosci. $2017 ; 11: 220$.

42. Haile Y, et al. Rab32 connects ER stress to mitochondrial defects in multiple sclerosis. J Neuroinflammation. 2017;14(1):19.

43. Ran FA, Hsu PD, Wright J, Agarwala V, Scott DA, Zhang F. Genome engineering using the CRISPR-Cas9 system. Nat Protoc. 2013;8(11):2281-2308

44. Holley JE, Newcombe J, Winyard PG, Gutowski NJ. Peroxiredoxin V in multiple sclerosis lesions: predominant expression by astrocytes. Mult Scler. 2007;13(8):955-961. 